\title{
Image and video quality assessment using LCD: comparisons with CRT conditions
}

\author{
Sylvain Tourancheau, Patrick Le Callet, and Dominique Barba \\ IRCCyN, University of Nantes, \\ Polytech'Nantes, rue Christian Pauc, 44304 Nantes, France. \\ sylvain.tourancheau@univ-nantes.fr
}

\begin{abstract}
In this paper, the impact of display on quality assessment is addressed. Subjective quality assessment experiments have been performed on both LCD and CRT displays. Two sets of still images and two sets of moving pictures have been assessed using either an ACR or a SAMVIQ protocol. Altogether, eight experiments have been led. Results are presented and discussed, some differences are pointed out. Concerning moving pictures, these differences seem to be mainly due to LCD moving artefacts such as motion blur. LCD motion blur has been measured objectively and with psychophysics experiments. A motion-blur metric based on the temporal characteristics of LCD can be defined. A prediction model have been then designed which predict the differences of perceived quality between CRT and LCD. This motion-blur-based model enables the estimation of perceived quality on LCD with respect to the perceived quality on CRT. Technical solutions to LCD motion blur can thus be evaluated on natural contents by this mean.
\end{abstract}

Keywords: subjective quality assessment, LCD motion blur, perception, measurements, displays

\section{Introduction}

In the recent years, subjective and objective quality assessment becomes a research topic of interest. Activities of the Video Quality Experts Group (VQEG) are a good example of this interest. Previous works [1] and work in progress [2] are mainly related to coding or transmitting purpose at a given resolution, e.g. coding artefacts and transmission errors. Display impact is rarely taken in account since for years only CRT were used and the distortions introduced by this technology were considered as insignificant with regards to other artefacts. However, since few years new display technologies have grown such as liquid crystal displays (LCD), plasma or projectors. These new technology are recent and not mature yet comparing to CRT, particularly for moving pictures. As a result, the perceived quality of images and moving pictures can be highly influenced by the display on which they are seen and knowledge about display should be used to improve quality assessment processes. Considering the whole chain, quality assessment should be able to manage dependency to other technology issues. LCD, for example, have many differences with CRT displays. Some subjective studies highlighted a high preference for CRT displays concerning moving pictures [3]. Many defects have been counted by viewers, such as colour differences, degradations in dark areas and de-interlacing artefacts for interlaced sequences. But among all these defects, motion blur seems to be the most annoying one, particularly in sequences with significant movements. An evidence for motion blur growing interest is the number of studies dealing with its measurement or its analysis $[4,5,6,7,8,9,10,11]$. Moreover, standardisation organisms such as TCO Development[12] and VESA [13] are currently working on requirements concerning this particular distortion. In this paper, some subjective quality assessment tests are described, as well with still pictures as with mov- 
ing ones. Both LCD and CRT displays are used and subjective scores are compared and discussed. In a second part, LCD motion blur is presented. Psycho-physics experiments and physical measurements are presented. Finally, an example of objective metric based on display knowledge is addressed. This prediction model is based on LCD motion blur and permits to estimate the perceived quality on LCD from the perceived quality on CRT.

\section{Subjective quality assess- ment experiments}

In this section, some subjective quality assessment experiments are described. They have been performed on both CRT and LCD displays, and considering still images as well as moving pictures.

\subsection{Image and video materials}

Four sets of materials have been used in the subjective quality assessment experiments. Two sets named SP1 and SP2 contain only still images while two other sets, MP1 and MP2, contain moving pictures:

- SP1: the Toyama database, i.e. 14 colour pictures $(768 \times 512)$, coded with JPEG2000 using 6 compression ratio, and with JPEG using 6 compression ratio (196 pictures).

- SP2: 5 colour pictures $(1280 \times 800)$, distorted using mainly several combinations of downscaling followed by up-scaling operations (20 pictures).

- MP1: 10 HDTV sequences $(1920 \times 1080$ interlaced, YUV 4:2:2) of 10-second length from the European broadcasters SVT and Euro1080, coded with H.264 using 7 different bit-rates (80 sequences).

- MP2: 4 of the latter ones, rescaled in a SDTV-similar format $(960 \times 540$ interlaced $)$, and coded with H.264 using 6 different bitrates (24 sequences).

\subsection{Viewing conditions and ob- servers}

All subjective quality assessment experiments have been performed in a standardised room. Room illumination, chromaticity background, display settings and viewing distance have been set according to ITU recommendations BT.500-11 [14] and BT.710-4 [15]. These parameters have been adjusted, when needed, for different contents and displays used in the experiments. For example, viewing distance was set to $3 \mathrm{H}$ for HDTV content, $6 \mathrm{H}$ for SDTV content and $4 H$ for Toyama database images, where $H$ is the height of the displayed picture. For experiments using moving pictures, a HD player has been used, which enables the realtime playback of uncompressed HDTV content. In all experiments, the same displays has been used: a JVC DT-V 1910CG (CRT) and a Philips T370 HW01 (LCD).

Each observer participating in the experiments was first checked for colour blindness with Ishihara test and for visual acuity with Monoyer's plates. People with at least one error in Ishihara's test or less than 9/10 in Monoyer's test were rejected. Observers'age ranged from 20's to 60's with an average around 30. Gender parity was respected as well as possible. The consistency of the individual scores was then evaluated after the tests have been completed by all the valid observers. It was done by applying a suitable rejection technique. This is a process in which all scores from a particular subject are omitted from the analysis of data. The number of retained observers depends on the protocol used (see 2.3).

\subsection{Protocols}

An absolute category rating (ACR) protocol has been used with the Toyama database (SP1 material) to follow the same test conditions as for experiments performed on CRT on a previous paper from Parvez et al. [16]. With this protocol, each picture is presented singly for subjective assessment and the observer is asked to provide his perception of the quality on a discrete scale that is divided into five numerical values and adjectives $(\operatorname{Bad}=1$, Poor $=2$, Fair $=3$, Good=4, and Excellent $=5$ ). The test presentation order is randomised differently for each observer. Following the VQEG recommenda- 
tions [2], at least 24 observers should be retained after the rejection process.

An effect of quantification of the subjective scores can appear with the ACR protocol and its discrete quality scale. In order to obtain more precise and more reliable results, we decided to use the SAMVIQ protocol for the three others sets of materials (SP2, MP1 and MP2). The SAMVIQ method [17] is a multi-stimuli continuous quality scale (MSCQS) protocol developed by France Telecom R\&D and standardised by the European Broadcasting Union (EBU) and by the International Telecommunications Union (ITU). It provides a precise and reliable measure of the subjective video quality as the observers have a random access to all sequences and can directly compare the impaired sequences among themselves and against the reference. This allows them to precisely build their assessment opinion. This is particularly interesting in a HDTV context where very little quality differences have to be identified [18]. Observers set the quality score using a continuous scale with a cursor moving from 0 to 100. Five adjectives were displayed on the scale to help the observers'choice $($ Bad $=10$, Poor $=30$, Fair $=50$, Good $=70$, and Excellent $=90)$. At least 15 observers should be retained after the rejection process.

\subsection{Results on still images (SP1 and SP2)}

\subsubsection{Toyama JPEG and JPEG2000 databases (SP1)}

The Toyama database has been assessed on CRT in the University of Toyama in Japan [16]. In order to compare both displays, we decided to perform the same subjective quality assessment using a LCD. However, design the same experiment in two different labs it's a real challenge and the so-called "lab effect" can occur. Actually, even if set as similar as possible, the viewing conditions can differ from on testing room to another. Furthermore, using two different pools of observers can also lead to slight differences. Also, some cultural differences can appear between France and Japan, in the way to assess quality. For example, the way that observers consider the adjectives on the quality ladder can be different. These adjectives were in English for the experiments in Japan, which was not the native lan-

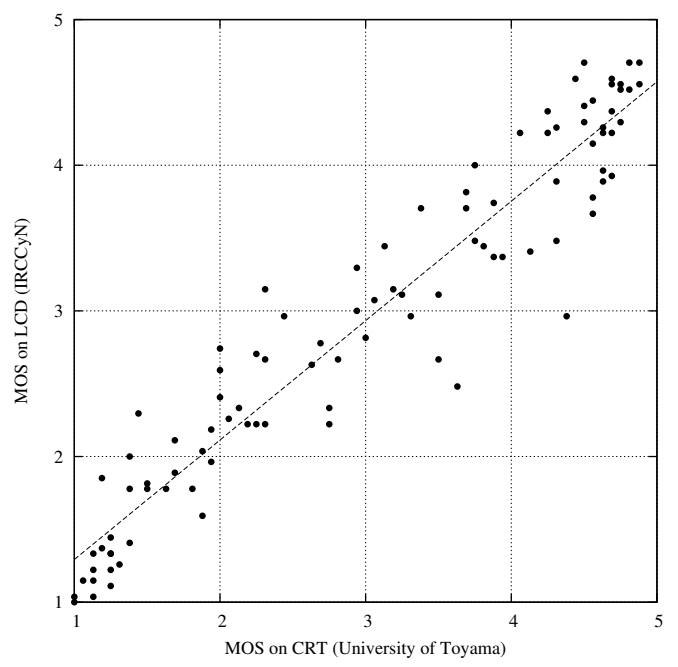

Figure 1: Comparison of MOS obtained on LCD (IRCCyN lab) and on CRT (University of Toyama lab) for the Toyama JPEG database.

guage of observers. They were translated in french for the experiments in France but the meaning of words can be slightly altered from one language to another.

Results between the two labs should be compared with regard to both the difference of displays and the lab effect. Figures 1 and 2 present the mean opinion scores (MOS) obtained on LCD in our lab as a function of MOS obtained on CRT in the University of Toyama [16] for the JPEG database and the JPEG2000 database respectively. In both cases, the set of scores obtained on CRT is are highly correlated with the one obtained on LCD, with linear correlation coefficients of 0.956 for the JPEG database and 0.966 for the JPEG2000 database. It's remarkable to have such a important correlation between experiments performed separately. However, when looking more precisely at the scores, a difference of scale can be observed. For both databases, the scores obtained on LCD (in France) are slightly better in the low part of the quality scale, whereas they are slightly lower in the high part of the quality scale. 


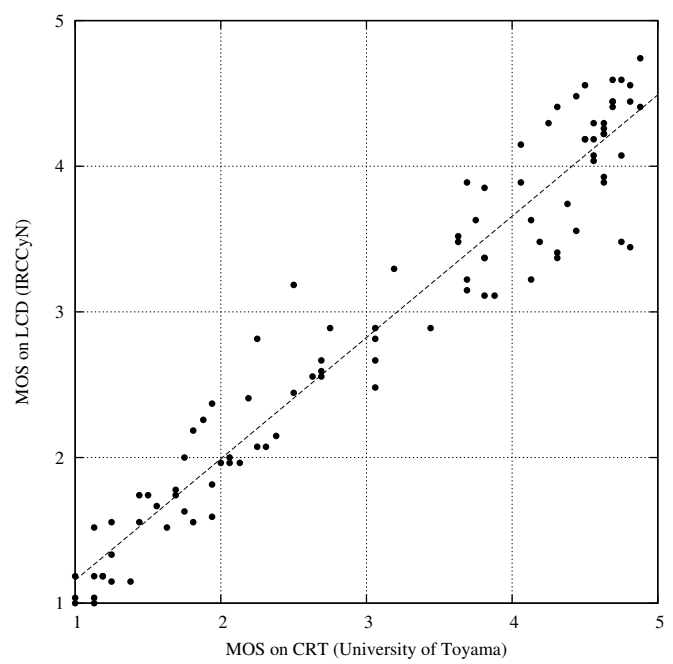

Figure 2: Comparison of MOS obtained on LCD (IRCCyN lab) and on CRT (University of Toyama lab) for the Toyama JPEG2000 database.

\subsubsection{HDTV format pictures (SP2)}

Images of SP2 set contain specific contents such as natural textures, flesh colours, oriented contours, water reflection, characters, etc. Each of them has been distorted by different combinations of a downscaling filtering following by an up-scaling filtering. They have been assessed on both LCD and CRT displays, in HDTV 1080i format $(1920 \times 1080$ in interlaced mode). Original and distorted pictures have been respectively inserted in a grey background to fit the format. It should be noticed that interlaced mode cannot be displayed on LCD, the screen thus performed a de-interlacing of the pictures in order to display them.

For this experiment, the same group of observers has been used for the two displays. The group has been split in two parts: the observers of the first part have passed the test on CRT first, the observers of the second part have passed the test on LCD first. In order to study the quality differences due to display technology, we focused on the MOS obtained for the hidden reference (non distorted) pictures. Results and $95 \%$ confidence intervals are shown in Table 1 . The difference of perceived quality $\triangle \mathrm{MOS}$ is computed as the difference between the scores obtained on CRT (MOS $\mathrm{MRT}_{\mathrm{CR}}$ ) and those obtained on LCD $\left(\mathrm{MOS}_{\mathrm{LCD}}\right)$ :

\begin{tabular}{|c|c|c|c|}
\hline Pictures & MOS $_{\text {CRT }}$ & MOS $_{\text {LCD }}$ & $\Delta$ MOS \\
\hline \hline FOOTBALL & $70.6(7.4)$ & $79.7(7.3)$ & -9.1 \\
HAND & $75.8(7.4)$ & $79.3(7.2)$ & -3.5 \\
HOUSE & $59.8(11.1)$ & $80.3(11.1)$ & -20.5 \\
LANDSCAPE & $75.8(5.8)$ & $75.7(5.4)$ & 0.1 \\
MAP & $59.8(10.9)$ & $83.9(10.7)$ & -24.1 \\
\hline
\end{tabular}

Table 1: Mean opinion scores and 95\% confidence intervals (between brackets) obtained on both displays for the set SP2 displayed in 1080i.

$$
\Delta \mathrm{MOS}=\mathrm{MOS}_{\mathrm{CRT}}-\mathrm{MOS}_{\mathrm{LCD}}
$$

It can be observed that for still pictures the quality on LCD is globally preferred. Overall, shortcomings of CRT displays such as flickering and limited range of luminance seems to lead to a lower feeling of natural and sense of immersion. LCD is brighter, vivid and colourful and the perceived quality of still pictures is clearly higher on it $\left(\Delta \mathrm{MOS}_{\text {mean }}=-11.4\right)$. On the whole set of 20 pictures (hidden reference and distorted ones), the linear correlation coefficient is 0.832 . As expected, quality scores on both displays are correlated but not so much. This could be due to the fact that some processing artefacts are more visible on one display than on the other.

\subsection{Results on moving pictures (MP1 and MP2)}

\subsubsection{HDTV sequences (MP1)}

Figure 3 shows the mean opinion scores obtained on LCD as a functions of those obtained on CRT. The linear correlation coefficient between the two sets of scores is 0.9487 . It can be seen that the perceived quality is globally better on CRT than on LCD. The average value of $\triangle \mathrm{MOS}$ over the 80 sequences is $\Delta$ MOS $_{\text {mean }}=10.2$. In order to study more precisely the impact of display without coding artefacts considerations, we can focus on the MOS of the hidden reference sequences presented in Table 2. It's interesting to notice that this loss of quality is quite important for sequences with quick movements such as Show, Concert, Foot and Voile. This loss of quality on LCD seems to be related to the quantity and/or the fastness of the movements in the sequence. 


\begin{tabular}{|c|c|c|c|}
\hline Sequences & MOS $_{\mathrm{CRT}}$ & MOS $_{\mathrm{LCD}}$ & $\Delta$ MOS \\
\hline \hline VOILE & $86.1(7.7)$ & $76.6(7.2)$ & 9.5 \\
FOOT & $83.3(6.4)$ & $74.1(7.6)$ & 9.2 \\
CONCERT & $82.6(7.2)$ & $73.1(4.3)$ & 9.5 \\
SHOW & $84.6(5.3)$ & $72.6(7.5)$ & 12.0 \\
CREDITS & $84.3(6.2)$ & $76.3(7.2)$ & 8.0 \\
MOBCAL & $79.9(6.1)$ & $80.6(5.7)$ & -0.7 \\
PARKRUN & $87.6(4.2)$ & $81.3(6.1)$ & 6.3 \\
SHIELDS & $85.3(3.7)$ & $76.3(7.3)$ & 9.0 \\
STOCKHOLM & $85.4(5.5)$ & $80.4(6.6)$ & 5.0 \\
GOLF & $77.5(5.8)$ & $78.5(6.1)$ & -1.0 \\
\hline
\end{tabular}

Table 2: Mean opinion scores and 95\% confidence intervals (between brackets) obtained on both displays for the set MP1.

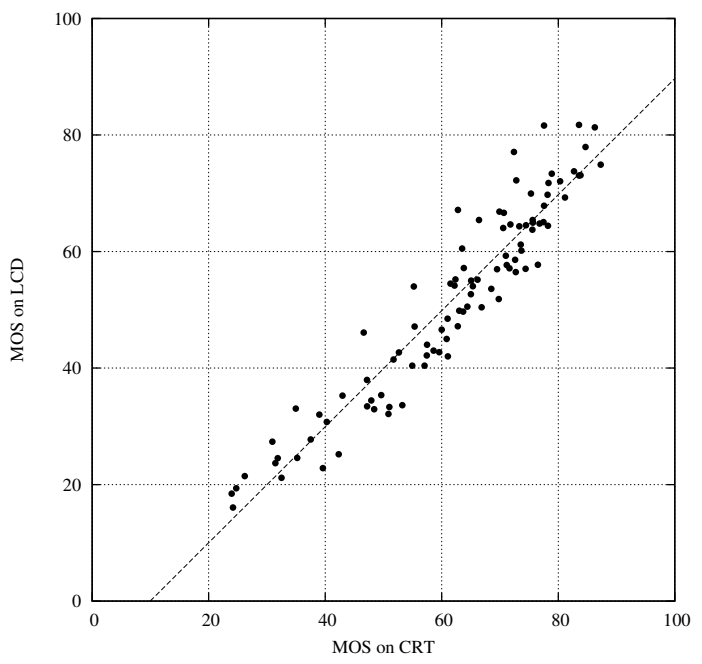

Figure 3: MOS obtained of LCD as a function of MOS obtained on CRT for the set MP1.

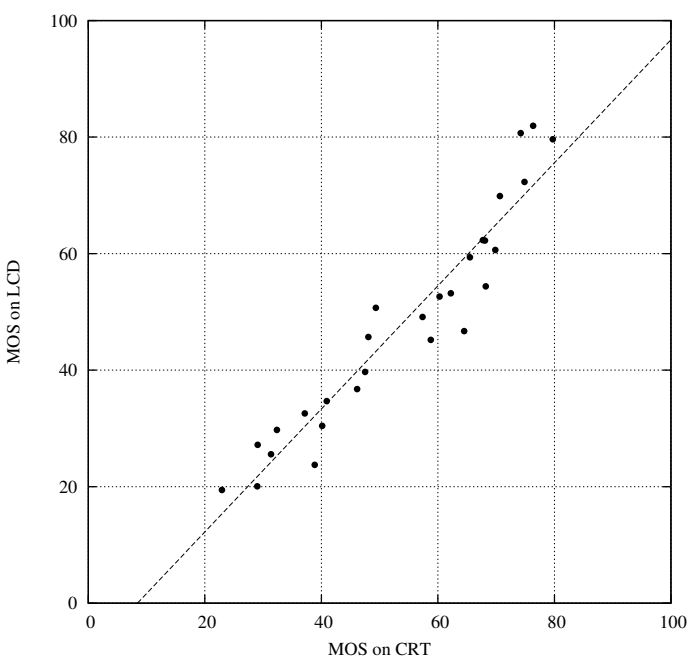

Figure 4: LCD MOS as a function of CRT MOS for the set MP2.

\subsubsection{SDTV sequences (MP2)}

The SDTV sequences of the set MP2 are computed from four HDTV sequences of the set MP1 through a half-band filtering followed by a down-sampling operation by a factor of 2 (both along horizontal and vertical directions). This processing is performed on each field of the interlaced HDTV sequence. The resulting 540i sequences are an approximation of the actual SDTV whereof format is $576 \mathrm{i}$, with the advantage that it does not necessitate any interpolation.

The MOS obtained on LCD are plotted as a function of the MOS obtained on CRT in Figure 4. The linear correlation coefficient between the two sets of scores is 0.9541 . Here again, the perceived quality is preferred on CRT than on LCD. But whereas the gap is quite important in HD (set MP1), it's less blatant for SD materials (set MP2). The average loss of quality between CRT and LCD is almost reduced by a factor $2: \Delta \mathrm{MOS}_{\text {mean }}=5.9$.

\subsection{Conclusion}

For all experiments, a high correlation exists between perceived quality on CRT and perceived quality on LCD. Experiments with Toyama database (SP1) have highlighted a difference in the use of the quality scale between CRT (Japan) and LCD (France). This result can be due to the dif- 
ference of display but it's likely that the lab effect (along with cultural differences) could be responsible for an important part, even if viewing conditions in the two labs were as close as possible.

Experiments with the set SP2 have shown a significant preference for images displayed on LCD whereas experiments with the set MP1 pointed out that moving pictures were largely preferred on CRT display. These results seem to agree with previous studies [3], highlighting that LCD moving artefacts could be responsible for a loss of perceived quality on LCD. More particularly, LCD motion blur can be very annoying on sequences with a lot of movements. Experiments with SDTV materials (set MP2) have also shown a difference of perceived of quality between CRT and LCD. But this difference was less significant than for HDTV materials.

In the following, the assumption is done that the loss of quality on LCD is mainly due to motion blur. In order to verify this hypothesis, the motion blur is analysed and measured. A linear model is validated which permits to predict blur from the temporal response function of the LCD.

\section{$3 \quad$ LCD motion blur}

\subsection{LCD motion blur perception}

Despite recent improvements to LCD technology such as response time compensation [19], LCD motion blur remains very annoying for sequences with rapid movements. In fact, even if the response time of a liquid crystal matrix was reduced to zero, motion blur would still appear. This is due to sampleand-hold behaviour of the display : the light intensity is sustained on the screen for the duration of the frame, whereas on CRT light intensity is a pulse which fades over the frame duration (cf. Figure 5). LCD displays are so called hold-type displays. The main difference happens when the eye of the observer is tracking a moving object on the screen: for a given frame, the picture is still on the screen while the eye is still moving slightly anticipating the movement of the object. Edges of this object are displaced on the retina resulting in a blur [20].

Several authors have analysed the motion blur perception $[20,21,7,8,9]$. Pan et al. [6] have developed a mathematical model in which the temporal response of the liquid crystal display is a parameter.

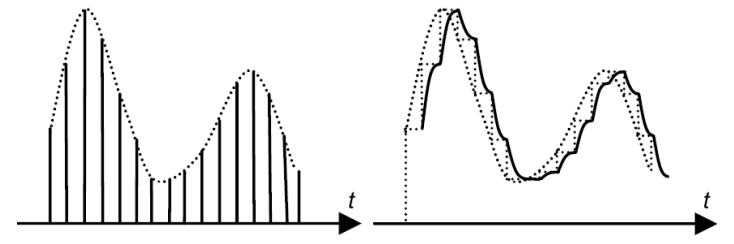

(a)

(b)

Figure 5: Temporal evolution of a pixel's intensity on a CRT display (a), on an LCD display (b). (from Pan et al. [6])

This model is designed to predict the perception of a moving edge on a LCD device. They showed that the perceived blurred edge width $B E W$ can be expressed as a function of the velocity $V$ of the object with the following relation:

$$
B E W=a V
$$

where $a$ can be obtained from the temporal response function of the LCD.

\subsection{Objective motion blur measure- ments}

Spatial measurement of a moving edge have been performed using a high-frame-rate stationary camera. The blurred edge profile is obtained from the spatial measurements by motion compensation and temporal integration $[11,10]$ to reproduce the smooth pursuit and the temporal integration of the eyes. This method is very similar to the famous MPRT measurement system [4] but it does not require a moving camera system and it's easier to carry out.

The apparatus used for this measurements consisted in a high-frame-rate CCD camera and a PC used to control the camera, to store grabbed frames, and to display stimuli on the test display. A JAI PULNiX's Gigabit Ethernet CCD camera, the TM-6740GE, has been used for these measurements. It was linked to the control PC via Ethernet, using a Gigabit Ethernet Vision (GigE Vision) interface which permits to reach high frame rate. Its frame rate has been set to $1200 \mathrm{~Hz}$ with a resolution of $224 \times 160$ pixels. The display frequency has been set to $60 \mathrm{~Hz}$, thus we obtain 20 CCD frames for each display frame. The distance between the measured display and the camera has 


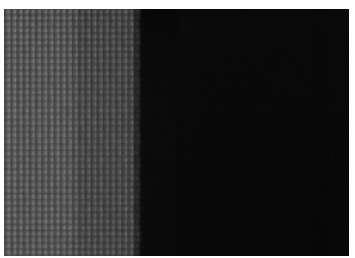

(a) Frame 0

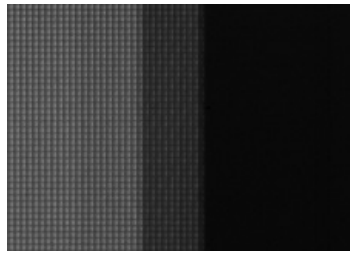

(c) Frame 8

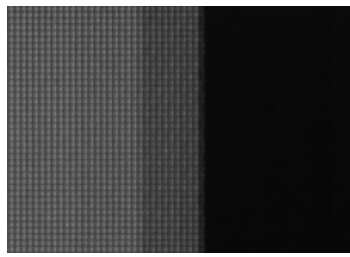

(e) Frame 16

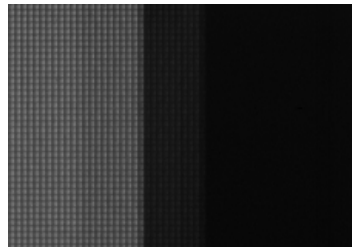

(b) Frame 4

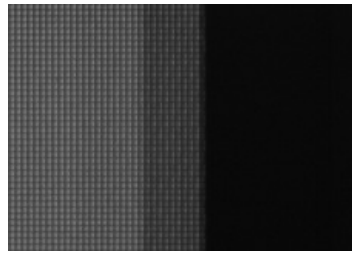

(d) Frame 12

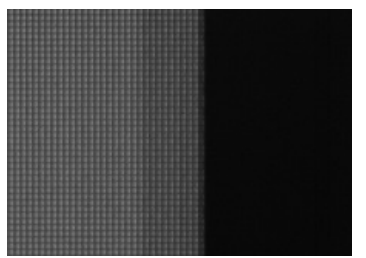

(f) Frame 20
Figure 6: CCD camera frames $(224 \times 160$ pixels $)$ taken during one period of the display frame rate. been set in such a way that one pixel on the display array is pictured by $4 \times 4$ pixels on the CCD array. This permitted us to obtain a good approximation of the $56 \times 40$ pixels display frame by computing the mean of each $4 \times 4$ blocks in the CCD frame. Stimuli were generated with Matlab on a PC using the PsychToolbox extension [22]. They consisted of a straight edge moving from left to right. One example of frames grabbed by the CCD camera is shown in Figure 6. As mentioned before, the blurred profile was obtained by motion compensation of each CCD frames to simulate the smooth pursuit of the eyes. The high camera frame rate and the precise calibration of apparatus to have $4 \times 4$ CCD camera pixels to picture one display pixel permit us to achieve this motion compensation precisely. Next, all frames are added to each other to simulate the temporal integration on the retina. An example of blurred edge obtained with this method is shown in Figure 7 for a edge moving with a velocity $V=10$ pixels per frame. The blurred edge width $B E W$ (in pixels) is measured as illustrated. The blurred edge time $B E T$ (in seconds or in frames) is generally used, it's expressed by dividing $B E W$ by the velocity $V$ (in pixels per seconds or pixels per frame):

$$
B E T=B E W / V
$$

Moreover, it has been observed that for a given grey-to-grey transition (i.e. for a given temporal response of the liquid crystal cells), BET was not varying with the velocity $V$. In other terms, the measured blur width $B E W$ was proportional to the velocity of the moving edge. This result agree with the relation 2 and the parameter $a$ can then be identified with the blurred edge time $B E T$.

\subsection{Perceived motion blur measure- ments}

Experiments have been designed in order to subjectively measure the perceived blur width as a function of motion speed. However, the perception of motion blur is directly related to the tracking of the moving object. If the observer stops to track the stimulus, to measure blur for example, then the blur is not perceived anymore. That's why we had to design an experiment in which the measurement of the blur is done while perceiving it. This has been done by displaying on LCD a periodical struc- 
IIIIIIIII

(a)

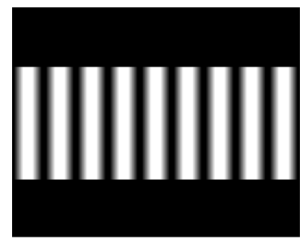

(b)

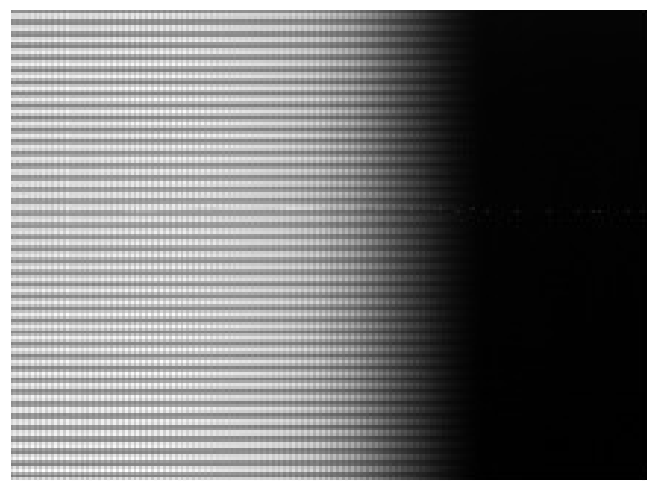

(a)

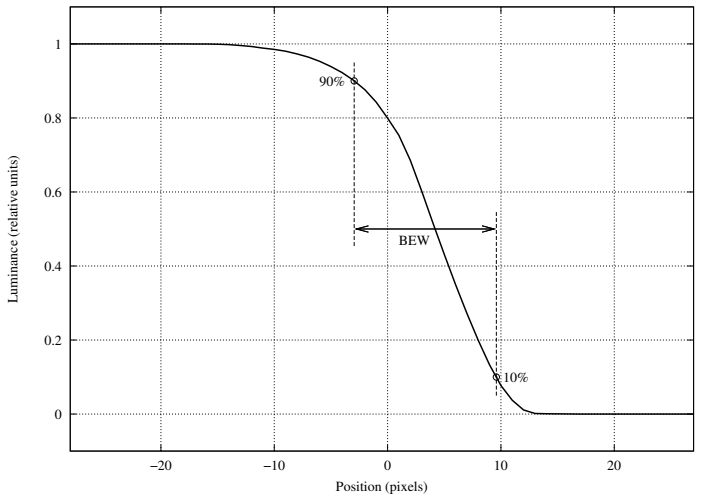

(b)

Figure 7: Blurred edge picture obtained after motion compensation of the camera frames and temporal summation (a), and blurred edge profile extract from the latter (b). Measurements have been performed for a black-to-white edge moving from left to right with a velocity of $V=10$ pixels per frame.
Figure 8: Displayed (a) and perceived (b) stimulus for an horizontal movement from left to right.

ture of bars moving at a constant speed $V$. Due to LCD motion blur, edges of the bars did not appear sharp as shown in Figure 8a but spread in the gap between two bars as in Figure 8b. During the test, the observer increased or decreased, in real-time, the space between the bars until he considered that the two blurred areas were just merging.

Many grey-to-grey transitions have been tested in both horizontal and vertical directions. The length of a session varied from an observer to an other, but the average time was between 10 and 15 minutes. Seven observers participated in the experiment, each has repeated the test twice, on different days. We finally obtained a set of 14 observations for each stimulus.

An example of results is shown in Figure 9 for a given grey-to-grey transition. In the explored range of speed, the width of blur $B E W$ was proportional to motion velocity $V$, as well for horizontal movement as for vertical movement. It can be observed that there were no significant differences between the two directions. For each grey-to-grey transition tested during the experiment, the same behaviour occurred and subjective results can be fitted by the linear relation 2 with a very high correlation coefficient. It was of 0.9984 for the transition illustrated in Figure 9.

\subsection{Conclusion}

Both objective and subjective measurements of LCD motion blur have led to a linear relation between the blurred edge width $B E W$ and the velocity $V$. The proportionality factor $a$ is constant for a given grey-to-grey transition. It has been identified to the blurred edge time $B E T$ and can be computed from the temporal response function of the LCD display $[9,11]$. This model enables the 


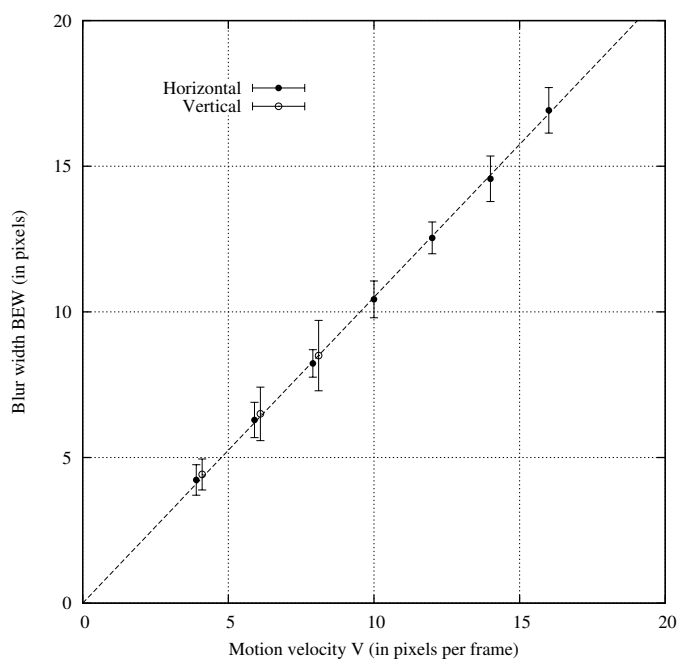

Figure 9: Perceived blur width BEW as a function of motion velocity $\mathrm{V}$, for a horizontal direction (filled circles) and for a vertical direction (open circles), with confidence intervals over seven observers.

prediction of perceived blur for a given velocity.

With regard to this result, the fact that the loss of quality on LCD was lower with SDTV material (see section 2) can be easily explained. In SDTV, the velocity of moving objects is reduced by two in terms of resolution (pixels per second) with respect to HDTV. The magnitude of perceived motion blur is then reduced in the same proportions. The loss of quality on LCD for SDTV materials $\left(\Delta \mathrm{MOS}_{\text {mean }}=5.9\right)$ was around two times lower than in HDTV $\left(\Delta \mathrm{MOS}_{\text {mean }}=10.2\right)$. This is another evidence to the assumption that LCD motion blur is the main cause of the loss of perceived quality on LCD. Moreover, this tends to show that LCD motion blur is typically an HDTV artefact since it's less visible and then less annoying at lower resolutions.

In the following section, a objective motion-blurbased model is designed in order to predict LCD perceived quality.

\section{Prediction model of video differences between CRT and LCD}

Figure 10 illustrates the both subjective and objective way to obtain the difference of quality $\triangle$ MOS. Since subjective assessment gives a value of $\triangle \mathrm{MOS}$ for each sequence, an objective model is designed in order to predict this quality loss $\Delta \mathrm{MOS}_{p}$ from the magnitude of blur in the sequence. As shown in the previous section, the perceived blur is linearly related to the velocity of the movement.

The prediction is made in four steps. Firstly, a motion estimation is performed along the sequence. This leads to the construction of tubes which are the sets of blocks positions along the direction of motion. Each tube is then classified according to his spatial content. Secondly, tubes categorised as textures and contours are selected and an average motion vector is computed from all the vectors of these selected tubes. Thirdly, an average magnitude of motion blur is predicted using the linear model of the previous section. Fourthly, $\Delta \mathrm{MOS}_{p}$ is computed from a prediction model.

\subsection{Motion estimation}

As sequences are interlaced, motion estimation is made on each field. A block $16 \times 8$ of an odd (resp. even) field is simultaneously compared to blocks of the two previous and the two next odd (resp. even) fields (Figure 11). The position which minimises the mean square error is chosen. Thus, a vector is obtained for each $16 \times 8$ block of each group of five consecutive odd (resp.even) fields. For each group of five frames, the motion vectors of even and odd fields are then merged in order to obtain a vector for each $16 \times 16$ block. These blocks which are followed along five frames are so called spatiotemporal tubes. Each tubes are classified into categories: contours, textures or uniform areas.

\subsection{Average blur index}

Since motion blur is only visible with a sufficient contrast [23], tubes classified contours and textures are selected. For each group of five frames, a spatial vector is computed averaging the vectors of selected tubes. These spatial vectors are then tempo- 

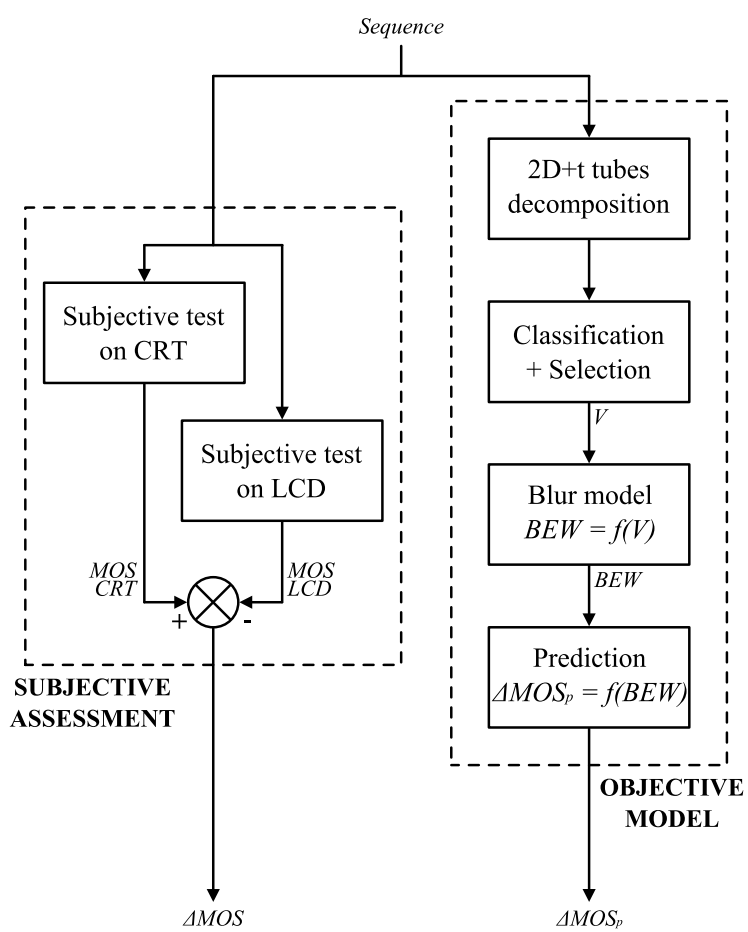

Figure 10: Diagram illustrating the objective prediction of the loss of quality $\Delta \mathrm{MOS}_{p}$.

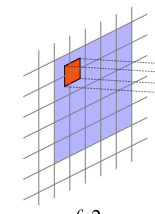

$f-2$

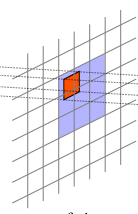

$f-1$

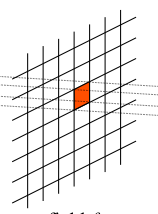

field $f$

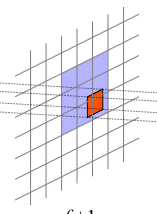

$f+1$

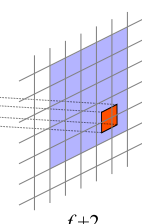

$f+2$
Figure 11: Construction of the spatio-temporal tubes. rally averaged along the sequence. A global motion vector is obtained for each sequence. The norm $V$ of this global vector is finally used to compute the width of perceived motion blur according to previous section (cf. Equation 2). The obtained $B E W$ is an indicator of the magnitude of perceived blur along the sequence.

\subsection{Prediction}

A indicator of the quantity of perceived motion blur has been computed for each sequence. The main objective of this work is to determine the relation between the LCD motion blur and the loss of quality observed between CRT and LCD displays. A non linear function $\Delta \mathrm{MOS}_{p}=f(B E W)$ has been constructed in order to predict the quality difference $\triangle$ MOS from the average blur quantity $B E W$. It has been assumed that this function has the following shape:

- In the first part $\left(B E W \leq B_{V}\right)$, the magnitude of perceived motion blur is too small to be noticeable and to influence quality perception.

- In the second one, the quality loss increases with magnitude of motion blur.

- Finally, in the third part, the quality difference saturates despite the increase of perceived blur $\left(B E W \geq B_{S}\right)$. This saturation may be due to contextual effects such as limited assessment scale and presence of quite distorted sequences during quality assessment.

The prediction model $\triangle \mathrm{MOS}_{p}=f(B E W)$ is represented in Figure 12. This model can be used to predict the quality loss $\triangle$ MOS between CRT and LCD from the average magnitude of blur measured on a sequence.

\subsection{Results}

The whole objective model presented in Figure 10 enables the prediction of the difference of perceived quality between CRT and LCD. This difference depends on the average motion blur measured on a sequence. This prediction of the loss of quality on LCD has sense when the motion blur is visible, i.e. for sequences with significant movements, and for video material with a high resolution. 


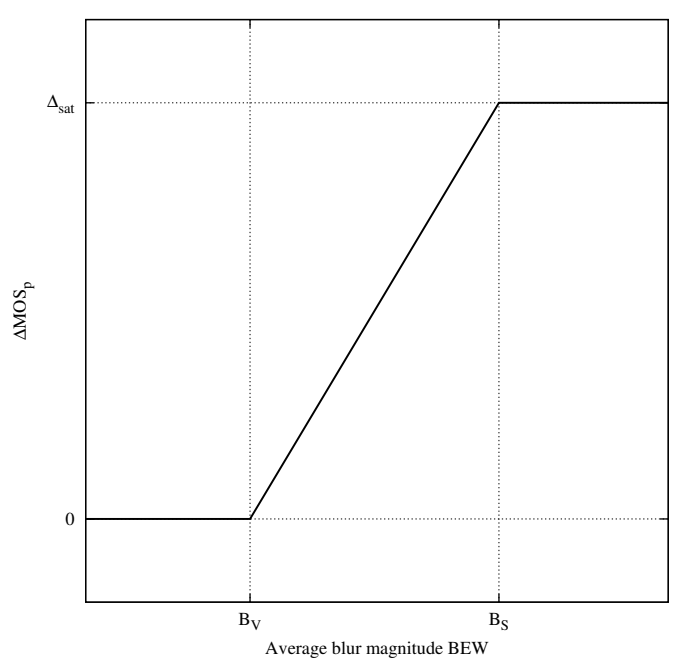

Figure 12: Prediction of the loss of quality $\triangle \mathrm{MOS}_{p}$ from the average blur magnitude $B E W$.

An estimation of the subjective quality scores on LCD from the subjective quality scores on CRT can be made using the following relation:

$$
\mathrm{MOS}_{\mathrm{LCD}}^{e s t}=\operatorname{MOS}_{\mathrm{CRT}}-\Delta \mathrm{MOS}_{p}
$$

This model is based on motion blur measurements, it can then be adapted to all types of temporal response. As a consequence, it permits to evaluate, on natural contents, the technical solutions to reduce motion blur such as back-light flashing or black data insertion.

\section{Conclusion}

In this paper, the impact of display on subjective quality assessment has been addressed. Subjective quality assessment experiments have been performed on both LCD and CRT displays. Two sets of still images and two sets of moving pictures have been assessed using either an ACR or a SAMVIQ protocol. Results have shown that CRT was globally preferred for moving pictures whereas it was the inverse for still images. As a consequence, the assumption has been done that the difference between display was mainly due to LCD moving artefacts such as motion blur. It should also be noticed that the difference of quality is reduced by a factor two for SDTV materials with respect to HDTV materials.

LCD motion blur has been then measured objectively and with psycho-physics experiments. It has been shown that the perceived blur width was varying linearly with the velocity. The parameter of this relation could be deduced from the temporal characteristics of the LCD display. This could explain the reduction of $\triangle \mathrm{MOS}$ between HDTV and SDTV materials.

Finally, a objective model has been designed which permits to predict the differences of perceived quality between CRT and LCD. This motion-blur-based model enables the estimation of perceived quality on LCD with respect to the perceived quality on CRT. Technical solutions to LCD motion blur such as back-light flashing or black data insertion can thus be evaluated on natural contents by this mean.

This study highlighted the fact that the recent LCD technology could lead to new visual artefacts when displaying moving pictures. These new artefacts are not significant at low resolutions, which validate the use of LCD for subjective video quality assessment in the Multimedia Test plan of the VQEG [2]. However, when increasing the resolution, they become more annoying and have an important impact on the perceived quality. It comes that the subjective video quality assessment at a high resolution (HDTV for example) should be led very carefully on LCD since a significant part of the perceived distortions could be due to the display. Liquid crystal displays have to be improved in order to reach the visual quality of CRT technology, particularly in a HDTV context. However, more reduced video applications supports such as laptop, cell phone, personal digital assistants, etc. are not as much affected due to their smaller display resolutions.

\section{Acknowledgements}

This study is supported by a student grant from the French région Pays de la Loire. Work presented in section 3.2 has been supported by TCO Development, Sweden. The authors would like to thank Arnaud Tirel and Romuald Pépion for their assistance in performing the experiments described in the paper. 


\section{References}

[1] VQEG, "Final report from the video quality experts group on the validation of objective models of video quality assessment," Tech. Rep., VQEG, 2003. http://www.vqeg.org.

[2] VQEG, "Multimedia group test plan," Tech. Rep. Draft version 1.16, VQEG, February 2007. http://www.vqeg.org.

[3] ITU, "Report on results of comparative subjective picture quality assessment test between CRT and LCD," Questions ITU-R 95/6, 102/6, International Telecommunication Union - Radiocommunication Study Groups, August 2005.

[4] K. Oka and Y. Enami, "Moving picture response time (MPRT) measurement system," SID Symposium Digest of Technical Papers, pp.1266-1269, SID, May 2004.

[5] M.A. Klompenhouwer, "Temporal impulse response and bandwidth of displays in relation to motion blur," SID Symposium Digest of Technical Papers, pp.1578-1581, SID, June 2005.

[6] H. Pan, X.F. Feng, and S. Daly, "LCD motion blur modeling and analysis," IEEE International Conference on Image Processing, ICIP 2005., pp.21-24, September 2005.

[7] X.F. Feng, "LCD motion-blur analysis, perception, and reduction using synchronized backlight flashing," Proceedings of the SPIE Conf. Human Vision and Electronic Imaging XI, Electronic Imaging 2006, SPIE, January 2006.

[8] X. Li, X. Yang, and K. Teunissen, "LCD motion artifact determination using simulation methods," SID Symposium Digest of Technical Papers, pp.6-9, SID, May 2006.

[9] A.B. Watson, "The Spatial Standard Observer: A human vision model for display inspection," SID Symposium Digest of Technical Papers, pp.1312-1315, SID, June 2006.

[10] M.E. Becker, "Motion blur measurement and evaluation: From theory to the laboratory," SID Symposium Digest of Technical Papers, pp.1122-1125, SID, May 2007.
[11] X.F. Feng, H. Pan, and S. Daly, "Comparison of motion blur measurement in lcd," SID Symposium Digest of Technical Papers, pp.11261129, SID, May 2007.

[12] TCO, "TCO'06 Media Displays," TCOF1076 version 1.2, TCO Development AB, Stockholm, Sweden, 2006.

[13] VESA, "Flat Panel Display Measurements (FPDM2)," Tech. Rep. 2.0 Update, Video Electronics Standards Association, May 2005.

[14] ITU, "Methodology for the subjective assessment of the quality of television pictures," Recommendation ITU-R BT.500-11, International Telecommunication Union - Radiocommunication Sector, June 2002.

[15] ITU, "Subjective assessment methods for image quality in high-definition television," Recommendation ITU-R BT.710-4, International Telecommunication Union - Radiocommunication Sector, November 1998.

[16] Z.M.P. Sazzad, Y. Kawayoke, and Y. Horita, "Spatial features based no reference image quality assessment for JPEG2000," IEEE International Conference on Image Processing, 2007. ICIP 2007., pp.517-520, September 2007.

[17] EBU, "SAMVIQ - Subjective assessment methodology for video quality," Report by the EBU Project Group B/VIM, European Broadcasting Union, May 2003.

[18] J.L. Blin, "New quality evaluation method suited to multimedia context: Samviq," Proceedings of the Second International Workshop on Video Processing and Quality Metrics, VPQM'06, Scottsdale, January 2006.

[19] R.I. McCartney, "A liquid crystal display response time compensation feature integrated into an LCD panel timing controller," SID Symposium Digest of Technical Papers, pp.1350-1353, SID, May 2003.

[20] T. Kurita, "Moving picture quality improvement for hold-type AM-LCDs," SID Symposium Digest of Technical Papers, pp.986-989, SID, June 2001. 
[21] M.A. Klompenhouwer, "The temporal MTF of displays and related video signal processing," IEEE International Conference on Image Processing, 2005. ICIP 2005., pp.13-16, September 2005 .

[22] D.H. Brainard, "The Psychophysics Toolbox," Spatial Vision, vol.10, no.4, pp.433-436, 1997.

[23] J. Laird, M. Rosen, J. Pelz, E. Montag, and S. Daly, "Spatio-velocity CSF as a function of retinal velocity using unstabilized stimuli," Proceedings of the SPIE Conf. Human Vision and Electronic Imaging XI, Electronic Imaging 2006, Janvier 2006. 